\title{
Linking research and development in primary care: primary care trusts, primary care research networks and primary care academics
}

Gill Rowlands STaRNet London, Department of Community Health Sciences, St George's Hospital Medical School, London, UK, Tessa Crilly STaRNet London, HEReCOM, London, UK, Mark Ashworth, STaRNet London, Department of General Practice and Primary Care, GKT Department of General Practice, King's College London, London, UK, Joan Mager Richmond Primary Care Trust, UK, Catherine Johns NHS Directorate of Health and Social Care for London, UK, and Sean Hilton Department of Community Health Sciences, St George's Hospital Medical School, London, UK

In the UK, primary care groups and trusts (PCG/Ts), also known as primary care organizations (PCOs), are the organizations that will drive forward the primary care development agenda outlined by the government. We undertook a study to investigate the views of PCOs on the place of research in primary care development and their role as participants in the research process. The study population consisted of all PCOs in south London $(n=23)$. An initial structured questionnaire explored the organizations' perceived research needs, the potential of the primary care research community to meet those needs and their current research activity. This was followed by semistructured interviews during which the participants were asked to discuss their views on the place of research in their primary care development agenda and in particular how research might inform the development of clinical skills and services in the short and medium term. The third stage of the project was an action based workshop at which general practitioners (GPs) and primary care nurses developed research questions arising from the earlier stages of the study. The PCOs identified three key areas of research support: public health, health economics and nursing research expertise. Research support was needed to help them identify population health needs and to evaluate new models of care and shifting professional boundaries. In contrast to traditional primary care research, PCOs required rapid delivery of research results, particularly when these were used to inform the commissioning of services. Our study has highlighted that PCOs have a development agenda requiring rigorous research and evaluation to ensure the best outcomes for patients. The differing skills, roles and responsibilities of PCOs, PCRNs and academic departments show how partnerships might develop to further research and development in primary care.

Key words: primary care research networks; primary care trusts; research

\section{Introduction}

In the UK, primary care groups and trusts (PCG/Ts) (primary care organizations: PCOs) are charged with delivering improved primary care at

Address for correspondence: Dr Gill Rowlands, Director STaRNet London, Department of Community Health Sciences, St George's Hospital Medical School, 6th Floor, Hunter Wing, Cranmer Terrace, London SW17 0RE, UK. Email: growland@sghms.ac.uk a local level (Department of Health, 2000). ${ }^{1}$ Given the complexity and volume of the timetable faced by PCOs, there is a risk that the research needed to underpin this development may stall unless facilitated by the research community.

\footnotetext{
${ }^{1}$ PCOs have differing levels of responsibility for commissioning secondary care and delivering primary care services. Primary Care Trusts (PCTs) have more complex and mature organizational structures than Primary Care Groups (PCGs) and correspondingly greater responsibilities.
} 
Primary care research networks (PCRNs) were set up to address the lack of research capacity identified by the Mant report (Mant, 1997) and have achieved success in promoting multidisciplinary research relevant to primary care (Thomas et al., 2001). There is recognition of the potential for PCOs to raise the profile and impact of research that is rooted in local needs and priorities and which can readily translate into changed practice (Thomas et al., 2000). The central role of PCOs in research is emphasized by the research governance framework implemented by the Department of Health (Department of Health, 2001). This requires PCOs to be aware and supportive of all research being conducted within their areas; thus all PCOs, even those not leading on research, must be aware of the benefits and potential risks that research might bring to their patients.

Finally we know that research findings are often not implemented into clinical practice (Haines and Jones, 1994); one of the arguments put forward for involving PCOs in research has been that this might lead to better implementation of research findings (Thomas et al., 2000). In this study we combined structured questionnaire responses and qualitative interview analyses to determine research priorities together with an action research methodology (Hampshire, 2000) to support PCOs in implementing research arising from their own declared priorities.

\section{Method}

The plan of investigation is shown in Figure 1.

The first stage of the study was a cross-sectional structured questionnaire survey of all south London PCOs $(n=23)$. South London was chosen as it is the area covered by one PCRN (STaRNet London), has a large population with varying levels of deprivation and health need (Fitzpatrick and Jacobson, 2001) and hosts four departments and faculties of primary care.

A structured questionnaire was developed by a multidisciplinary research team composed of a PCO chief executive, three GPs, a health economist and a deputy head of research and development from the NHS Executive. The content of the questionnaire was piloted by primary care development staff in one PCO. The questionnaire addressed Primary Health Care Research and Development 2004; 5: 255-263 perceived research needs, the potential of the primary care research community to meet those needs, their current research activity and involvement with PCRNs. One copy was sent to the chief executive of each $\mathrm{PCO}$ with the request that it be forwarded to the person most appropriate to answer it. Each questionnaire was followed up by a faceto-face or telephone interview of the person who completed the form.

Results from the survey informed the second phase of the study in which a series of indepth semi-structured interviews of chief executives of the same PCOs were conducted by one of us (TC). The interviews took place between April and May 2001. By this date there were 21 PCOs; anticipated mergers reduced the number of interviewees to 18 as PCOs had made shared working arrangements with future partners.

Prior to the interview, each interviewee was sent a briefing paper describing the aims of the project and the interview schedule (see Appendix). The briefing paper built on the findings of the questionnaire; in particular the low levels of current research activity implied that PCOs might find it difficult to discuss research within their organizations; interview questions therefore focused on information and service needs. Interviewees were encouraged to discuss possible research opportunities arising from the areas in the interview schedule. Each interview was recorded and transcribed.

TC commenced her analysis by assigning topics raised during the interview alongside a précis of current/potential action and research and development opportunity identified by the respondent. While topics were common to more than one PCO, current/potential action and research and development opportunities were unique to each, amplifying the meaning and context of the topics within individual organizations. As the analysis progressed it became clear that many of the topics could be grouped into more general themes. These were more interpretative and analytical in nature, providing a narrative thread drawing the topics into a coherent set of development and information needs. TC and GR discussed and refined the emerging themes as the analysis progressed. The frequency with which each topic was raised was recorded, and the interview results were crosstabulated. Emergent themes were triangulated by feedback to the participants. 


\section{December 2000 to January 2001}

Cross sectional survey of south London PCOs $\mathbf{N}=\mathbf{2 3}$

\begin{tabular}{|c|}
\hline April to May \\
2001 \\
\hline
\end{tabular}

June 2001 to
October 2002

Qualitative study Indepth semi-structured interviews with iterative analysis $\mathrm{N}=18$ (involving $21 \mathrm{PCOs}$ )*
Action research stage

- development of research questions from themes identified by the PCOs

- production of research findings shared across south London 12 PCOs

* the number of south London PCOs had reduced from 23 to 21 through mergers

Figure 1 Plan of investigation

The third phase of this study took place two months later when representatives from the PCOs met as a group together with the research network and primary care academics. Each PCO was asked to nominate at least one clinician (doctor or nurse) who would want involvement in developing or undertaking research to attend.

The group developed research projects through an action research iterative process, based on presentation of the outcomes of the consultation exercise. A voting system was devised whereby each participant had three 'votes' which they could distribute as they saw fit between the themes identified from the earlier stages of the study. The themes attracting most votes were selected. These were then linked with clinical areas identified in the interviews. Research questions were then developed through small group work, one group for each theme. The primary care academics and PCRN members distributed themselves between each of the small groups to ensure academic input to each project.

\section{Results}

\section{Questionnaire survey results}

Results were obtained from 18 out of $23(78 \%)$ PCOs. At the time of the study four organizations were primary care trusts (PCTs) and 19 were primary care groups (PCGs). The questionnaire was completed by chief executives (14 PCOs), clinical director (a GP) (one PCO), PCG chairs (GPs) (two PCOs) and an audit/research and development lead (1 PCO). The five nonresponders were all PCGs. The most frequently reported research needs related to clinical effectiveness (implementing research findings, promoting evidence based medicine and health needs assessment: seven PCOs) and to the need to develop 
research skills and infrastructure (six PCOs). Three of the PCOs (two PCTs, one PCG) were actively involved in research; these all commented that the current research external funding process was too protracted to provide answers to research and development questions within PCO timescales. All the 15 responding PCOs not actively involved in research commented that they saw research skills and activity as desirable.

\section{Semi-structured indepth interviews}

All PCOs in south London agreed to participate. Eighteen interviewees represented 23 PCOs. PCOs were not asked about the place of research within the agenda of their organizations. However, eight $(44 \%)$ of participants did try to define research and development (R\&D) during their interviews; these found it difficult to differentiate between undertaking primary research, local evaluation of both local and national research findings and the role of R\&D in primary care. As expected from the questionnaire survey participants found it easier to define information and service needs rather than specific research requirements.

Thematic analysis generated eight themes arising from 36 topics (Table 1). These were as follows:

\section{Information needs}

All $(100 \%)$ of the interviewees referred to this theme. Twelve $(67 \%)$ of the participants talked about the need to explore health needs specific to their populations. Eleven $(61 \%)$ talked about the difficulties they had experienced collecting accurate GP and secondary care activity data and how this had made it difficult to plan for change. Potential research topics centred on the collection of accurate data and the most effective ways of using the data to inform and improve services.

\section{Managing the primary/secondary/social care interface}

Thirteen (72\%) interviewees talked about the need to address interface issues. Possible solutions were discussed such as managing demand, developing integrated patient care pathways and improving 'illness prevention'. Twelve $(67 \%)$ of participants talked about shifting roles within primary/secondary and primary/social care. They recognized the potential advantages of approaches that could improve care, reduce workload and increase job satisfaction, thus addressing recruitment and retention issues. Eight (44\%) did, however, express reservations about the potential impact of shifting boundaries on primary care workloads. Linked research needs focused on the impact on patients and practitioners of new methods of delivering care.

\section{Work roles and staffing within primary care}

This area was discussed by $12(67 \%)$ interviewees. The topics raised here mirrored the changes in primary/secondary/social care discussed above. Specifically, interviewees talked about changing boundaries between GPs, practice nurses and community therapists. The need for evaluation of the impact of these changes on patients and staff was highlighted, particularly concerning development of nursing roles.

\section{Clinical governance and sharing knowledge}

This topic arose in seven (39\%) interviews. Participants talked about the need to develop ways to assess those aspects of quality of care that are hard to measure objectively but which impact on patients:

It would be very refreshing to have something that tried to look at quality from a purely primary care perspective, which would not just measure the science but also sought to preserve the art.

(Clinical director of a PCG)

\section{Clinical areas}

Clinical areas were used as examples to illustrate the topics discussed. The clinical areas most frequently mentioned were ischaemic heart disease, cancer services, mental health, older people and diabetes.

\section{Care of older people}

Care of older people was classed as a 'theme' as well as a 'topic'. This was because it emerged as a key area in which interprofessional and interorganizational boundaries were changing rapidly, often with little or no research evidence on the impact of the changes on patients and health professionals. Several participants mentioned anxiety among the community that new models were driven by the search for cheaper rather than more effective solutions. 
Table 1 Main research themes and topics identified by PCOs (18 interviews)

\begin{tabular}{|c|c|c|c|}
\hline Theme & Frequency (\%) & Topics & Frequency (\%) \\
\hline Information needs & $18(100)$ & $\begin{array}{l}\text { Health needs assessment } \\
\text { Secondary/practice level commissioning } \\
\text { information } \\
\text { Practice-based clinical information } \\
\text { Information systems }\end{array}$ & $\begin{array}{l}12(67) \\
11(61) \\
5(28) \\
3(17)\end{array}$ \\
\hline $\begin{array}{l}\text { Managing the primary/ } \\
\text { secondary/social care interface }\end{array}$ & $13(72)$ & $\begin{array}{l}\text { Shifting boundaries within } \\
\text { primary/secondary/social care } \\
\text { Demand management } \\
\text { Access } \\
\text { Integrated care pathway } \\
\text { Secondary care resource shift }\end{array}$ & $\begin{array}{l}12(67) \\
7(39) \\
4(22) \\
5(28) \\
3(17)\end{array}$ \\
\hline Work roles and staffing & $12(67)$ & $\begin{array}{l}\text { Practice staffing } \\
\text { Primary care workload } \\
\text { Work roles } \\
\text { Subspecialists vs. generalists } \\
\text { GP specialists }\end{array}$ & $\begin{array}{r}2(11) \\
3(17) \\
11(61) \\
2(11) \\
9(50)\end{array}$ \\
\hline $\begin{array}{l}\text { Clinical governance and sharing } \\
\text { knowledge }\end{array}$ & $7(39)$ & $\begin{array}{l}\text { Clinical governance } \\
\text { Sharing knowledge }\end{array}$ & $\begin{array}{l}5(28) \\
2(11)\end{array}$ \\
\hline $\begin{array}{l}\text { Clinical areas (excluding care of } \\
\text { older people) }\end{array}$ & $14(78)$ & $\begin{array}{l}\text { Clinical areas (excluding care of older } \\
\text { people): } \\
\text { Children } \\
\text { Sexual health } \\
\text { Diabetes } \\
\text { Dermatology } \\
\text { Trauma and orthopaedics } \\
\text { Neurology } \\
\text { Nephrology } \\
\text { Care of the elderly/osteoporosis } \\
\text { CHD } \\
\text { Mental health } \\
\text { Cancer } \\
\text { NSF specialities } \\
\text { Public health } \\
\text { Audit and projects }\end{array}$ & $\begin{array}{ll}3 & (17) \\
3 & (17) \\
7 & (39) \\
1 & (6) \\
1 & (6) \\
1 & (6) \\
1 & (6) \\
2 & (11) \\
6 & (33) \\
6 & (33) \\
1 & (6) \\
8 & (44) \\
3 & (17) \\
1 & (6)\end{array}$ \\
\hline Services for older people & $10(56)$ & Services for older people & $10(56)$ \\
\hline $\begin{array}{l}\text { Promoting locally based research } \\
\text { and development (R\&D) }\end{array}$ & $9(50)$ & $\begin{array}{l}\text { R\&D principles and dissemination } \\
\text { Cost effectiveness/evidence base } \\
\text { Service developments and priority setting } \\
\text { Public health skills }\end{array}$ & $\begin{array}{l}8(44) \\
2(11) \\
7(39) \\
3(17)\end{array}$ \\
\hline Organizational structures & $8(44)$ & Organizational structures & $8(44)$ \\
\hline
\end{tabular}

Promoting locally based research and development

We found enthusiasm for developing research within PCOs, with a focus on the development of research skills and capacity. Participants wanted research to be locally based and to do it rather than have it done to them (PCT research and development lead). They saw the value of sharing resources; as one interviewee said ... 'we have all spent a fortune on pilots' (chief executive officer of a PCG). Participants talked about the need for specialist skills, especially in public health and health economics, to help them design, undertake and interpret their research. Research could thus address local health needs and be used to inform the development and commissioning of services. 
An example given by one PCG was sickle cell disease; local research could show whether new money would be better spent on pain management or psychological therapy and support for families.

\section{Organizational structures}

The climate of organizational change in which the PCOs found themselves led eight (44\%) of them to identify the evaluation of new organizational structures as a priority. They talked about the need to evaluate what they perceived as a drive towards larger and different units of delivery of care such as 'clusters' of practices and the widening of the 'classical' primary medical health care team to specialities such as dentistry. These PCTs discussed the need for both local and wider evaluation of alternative health care delivery structures.

The eight themes were fed back to each PCO representative. Participants said that the outcomes were a valid reflection of their thoughts on PCObased research. There was no modification of themes following this triangulation.

\section{The development of research questions from the research priorities identified by the PCOs}

Twelve of the 21 PCOs $(57 \%)$ participated in this stage of the project. In contrast with the earlier stages of the project involving senior management staff, PCOs were asked to identify primary care workers interested in developing and undertaking some research. Eighteen people attended from the 12 PCOs; eight GPs, six nurses (one district nurse, one walk-in centre nurse consultant, two health visitors, one nurse manager and one primary care researcher), and four administrators (two PCG primary care managers, one practice manager and one chief executive). Three topics were selected by the whole group for further research. These were demand management, health needs assessment and work roles. The resultant research projects are summarized in Table 2.

\section{Discussion}

South London covers a population of 2.7 million people. The inner city boroughs of south London have some of the highest levels of deprivation and amongst the worst infant mortality rates and lowest life expectancy rates in England (Fitzpatrick and Jacobson, 2001). The integration of research into the development agenda of south London PCOs is all the more pressing considering the health needs of this population.

\section{Main findings}

The questionnaire survey highlighted a mismatch between the information needs of PCOs and the volume of research addressing those needs, exacerbated by the pace of traditional externally funded, peer reviewed research. In the semistructured interviews the most prominent topics were interface issues, information needs and work roles. Although all the topics were discussed at the workshop following the individual interview, it was these three that provided the three research topic areas generated by the action research workshop.

\section{Limitations of this project}

For most of the PCOs the questionnaire and subsequent interviews were undertaken by the chief executives, with the minority completed by senior GPs and, in one case, by an audit/research and development lead (nonmedical). Although each was asked to report on the perspective of their own PCO, the findings are likely to have reflected the priorities of the respondents and might well have been very different if the GPs and nurses working within the PCOs had been surveyed. In contrast most of those involved in the action research stage of the project were clinicians nominated by their PCTs. However we feel that this pattern reflects the situation within PCOs whereby chief executives and senior management decide on local primary care development (and hence research) priorities whilst the clinicians develop and undertake the research itself.

The interview schedule was informed by our own experience and beliefs. Three of the research team are GPs whilst TC is a health economist. Since the interview schedule focused on information and clinical evidence needs it is not surprising that these are reflected in the study findings. The interview framework was, however, derived from the questionnaire and modified by the views of a PCT chief executive (JM). In addition, the preliminary findings were triangulated by checking back with the participants. Although the findings will have been influenced by the research team they appear to be a valid reflection of the views of PCOs. 
Table 2 Early research questions identified by the workshop

\begin{tabular}{lll} 
Research topic & Clinical area & Early research question \\
\hline Demand management & A\&E, care of older people & $\begin{array}{l}\text { How can PCOs predict patients' use of A\&E } \\
\text { services? }\end{array}$ \\
Health needs assessment & Older people & $\begin{array}{l}\text { What are the barriers to mobility for older people? } \\
\text { Work roles }\end{array}$ \\
Mental health & $\begin{array}{l}\text { How can skills in the detection and management of } \\
\text { depression in primary care be shared amongst } \\
\text { community nurses? }\end{array}$
\end{tabular}

The participants were not asked to rate the importance of research amongst their organizational priorities, although all obviously felt it important enough to participate in this piece of work. An exploration of the perceived importance of research within the organizations would have enabled a greater understanding of the context in which R\&D needs can be identified and the likelihood of such needs being translated into research and hence future development.

The action research stage of the project was designed and led by GR. The choice of subjects to develop into research questions will have been influenced by both the presentation of the interview outcomes and the method of selecting research question arising from them. It is likely that another set of attendees or the same attendees with different facilitators would have developed different research questions. The aim of the workshop was, however, to facilitate primary care doctors and nurses to develop research questions that had arisen from the survey; we feel this was achieved by the process we adopted.

\section{Further work}

This study looked at the research priorities of PCOs. Given the increasing role of PCOs within research in primary care it would be interesting to investigate the wider context of research within PCOs. In particular it would be valuable to understand the extent to which research is seen as 'integral to' rather than 'added on' to primary care development and the extent to which research findings are implemented into practice.

STaRNet London and members of the academic departments and faculties of primary care in south London are supporting the implementation of the three selected projects through the stages of research protocol development through to project completion, dissemination of results and translation of the findings into local practice. The action research stage of the study utilized only a small proportion of the potential research topics identified through the interviews. The full survey findings, in an anonymized form, are available from the lead author. Both the complete findings and the themes summarized in Table 1 could be used by other researchers to generate their own projects under these broad headings.

\section{Implications of this work}

We found that PCOs recognized the large amount of evidence they required to undertake their development agenda. Very few (three PCOs, $17 \%$ ) of the PCOs responding to the initial questionnaire were actively involved in research but all the participants recognized the need to develop research capacity and activity. The differing skills, roles and responsibilities of PCOs, PCRNs and academic departments show how partnerships might develop to further research and development in primary care.

PCOs have a central role in developing primary care services but those participating in our study showed a range of views about the nature and place of research in the agendas of their organizations. For maximum benefit to patients, development must be based on the findings from high quality research. This requirement is recognized in the Research Governance Framework (RGF) (Department of Health, 2001); PCOs must be aware of and supportive of all research undertaken in their localities. This study shows that PCOs are aware of their new responsibilities in research and development but are also aware of their current lack of research skills. An immediate 
role can be identified for PCRNs and academic departments of primary care in providing PCOs with research support. This study has shown that some PCOs can be directly engaged in research through an action research approach. We recognize that whilst this approach may not suit all PCOs it will ensure that they are able to become involved in the research process at the level that suits their needs. Thus 'innovators' (Haines and Jones, 1994) can develop skills and undertake primary research whilst wider dissemination of research findings and development of critical appraisal skills will ensure that a research culture of benefit to patients and clinicians can develop in all PCOs.

\section{Implications for primary care research networks}

Close links between networks and PCOs should ensure that research is important and relevant to patients and will enable PCRNs to facilitate the development of skills in the community. Networks also have a role in facilitating the sharing of research ideas, skills and time to avoid duplication of effort between PCOs and to share outcomes quickly across their localities.

\section{Implications for those funding research and PCRNs}

From the perspective of a $\mathrm{PCO}$, commissioning procedures for research can appear to be cumbersome, bureaucratic and unresponsive to the needs of the service. It is important that PCOs are able to inform and influence the priority setting agenda for NHS research and development nationally and locally. There has been success at regional level with programmes like the NHS London Regional Office R\&D Directorate Primary Care Studies Research Programme. This enabled PCOs to identify important research priorities, with London PCRNs being instrumental in facilitating the success of this programme. Those responsible for commissioning research under the latest NHS Executive reorganization need to retain this local flexibility in combination with ensuring that all research meets the rigorous quality standards in the Research Governance Framework (Department of Health, 2001).

\section{Implications for academic departments of primary care}

Research (including the development of new researchers through support and supervision) is a core function of academic departments. There is a tension between the current assessment of the research output of universities and the need for departments to support PCO-led research. Such research, locally focused and short term, is less likely to generate external peer reviewed grant income and publication than 'classic' academic research. The PCOs need the support and skills of academic departments of primary care to ensure that their research is of the highest standards; departments may be unable to provide such support unless they are able to gain appropriate recognition for doing so.

\section{Implications in an international context}

Whilst PCG/PCT PCOs are currently unique to the UK there are parallels with systems such as the health maintenance organisations (HMOs) in North America (Miller, 1997) where primary care services are developing more central 'gatekeeper' functions in health care provision. In any primary care-led health system the research priorities of the organizations delivering primary care will impact upon patient care and on the balance of research undertaken between the primary and specialist health care providers. It would be interesting to compare the research priorities of the emerging North American HMOs with those of our developing PCTs.

\section{Linking primary care research and development}

The challenge in the new NHS is for all those involved in commissioning and undertaking research, implementing evidence-based practice and delivering the service to share the task of ensuring that primary care development is underpinned by rigorous research. This study describes one approach that has identified PCO research needs and supported PCOs to start to generate the information they need to answer some of their questions. The challenge for PCRNs and academic departments of primary care is to ensure that the information produced is rigorous, generalizable and disseminated; the challenge for PCOs is to facilitate the implementation of these research findings into primary care development (Haines and Jones, 1994). 


\section{Acknowledgements}

We would like to thank all the south London Primary Care Groups and Trusts employees who gave their time to this study. This project was commissioned and funded by STaRNet London. STaRNet London is supported and funded by the NHS Directorate for Health and Social Care (London Office). The study was devised and developed by GR, MA, JM and CJ with advice from SH. MA led and analyzed the questionnaire survey. The interviews were conducted and analyzed by TC with support from GR. The workshop was led by GR, MA and TC. The paper was written by GR with contributions from all the other authors.

\section{References}

Department of Health. 2000: The NHS Plan. A plan for investment. A plan for reform. London: HMSO.

Department of Health. 2001: Research governance framework for health and social care. London: HMSO.

Fitzpatrick, J. and Jacobson, B. 2001: Mapping health inequalities across London. London: London Health Observatory.

Haines, A. and Jones, R. 1994: Implementing findings of research. British Medical Journal 308, 1488-92.

Hampshire, A.J. 2000: What is action research and can it promote change in primary care? Journal of Evaluation in Clinical Practice 6(4), 337-43.

Mant, D. 1997: $R \& D$ in primary care. National Working Group Report, NHS Executive. London: HMSO.
Miller, T.E. 1997: Managed care regulation. Journal of the American Medical Association 278(13), 1102-109.

Thomas, P., Griffiths, F., Kai, J. and O'Dwyer, A. 2001: Networks for research in primary health care. British Medical Journal 322(7286), 588-90.

Thomas, P., Kai, J., O'Dwyer, A. and Griffiths, F. 2000: Primary care groups and research networks: opportunities for R\&D in context. British Journal of General Practice 50(451), 91-92.

\section{Appendix 1: Semi-structured interview} schedule

1. What information do you need to inform your commissioning process in the short term (results in one year)?

2. What information do you need to improve clinical practice in the short term (again results within a year)?

3. What specialities do you identify as key new players in the next one to three years? Can you see a role for making links through shared research?

4. What about service development and clinical evidence needs in the medium term (results in three to five years)?

Individuals were encouraged to think about their organization's information and development needs and how research might inform that process.

Copies of the full briefing documents are available from the lead author. 